\title{
Ulnar Shortening Osteotomy: Are Complications Under Reported?
}

\author{
Samuel K. L. Chan ${ }^{1} \cdot$ T. Singh ${ }^{1} \cdot{\text { R } \operatorname{Pinder}^{1} \cdot \text { S. } \operatorname{Tan}^{1} \cdot \text { M. A. Craigen }}^{1}$
}

Received: 3 June 2015 / Accepted: 14 September 2015 / Published online: 23 September 2015

(C) Society of the Hand \& Microsurgeons of India 2015

\begin{abstract}
Ulnar shortening osteotomy is an accepted treatment for ulnar impaction syndrome and numerous techniques for have been described in the literature. We present our single surgeon case series over 10 years of oblique ulnar shortening osteotomy using a jig technique. A review of the current literature with reference to the risk of complications is included. All patients undergoing ulnar shortening osteotomy from 2001 to 2011 were identified and case-notes were reviewed retrospectively. Risks of complications included metalwork irritation (51\%), non-union (6.3\%), refracture $(1.6 \%)$ and chronic regional pain syndrome (1.6\%). Oblique ulnar osteotomy using a jig technique is reliable and reproducible in shortening the ulna and maintaining alignment, rotation and compression at the osteotomy site. Symptoms improved in the majority of patients. However, the morbidity associated with the procedure may be underestimated as highlighted by our complication rates. Surgeons should counsel patients appropriately when offering ulnar shortening osteotomy.
\end{abstract}

Keywords Ulnar impaction syndrome $\cdot$ Ulnar shortening osteotomy $\cdot$ Complications

\section{Introduction}

Ulnar impaction syndrome (UIS) was first described by Friedman and Palmar as a clinical condition characterised by ulnar wrist pain, swelling and limitation of motion due to

Samuel K. L. Chan

samuelchan@doctors.org.uk

1 Royal Orthopaedic Hospital NHS Foundation Trust, Bristol Road South, Birmingham B31 2AP, UK excess load bearing of the ulna head against the triangular fibrocartilage complex (TFCC) and ulnar carpus [1]. With continued impaction, progressive damage occurs including TFCC tears, ulno-carpal chondromalacia, lunotriquetral ligament tears, and ulnocarpal osteoarthritis [1]. These can either be secondary to distal radius fracture malunion, premature physeal arrest, radial head excision, Essex-Lopresti injury, or due to primary or idiopathic UIS.

Ulnar shortening osteotomy is an accepted treatment for a variety of ulnar sided wrist disorders. It is most commonly used for UIS but has also been used in the treatment of distal radioulnar joint (DRUJ) disorders [2-4], TFCC injuries [5-8], and ulnocarpal instability [9]. The basis of treatment with ulnar shortening for UIS is mechanical decompression of the ulnocarpal joint by transferring load to the radiocarpal joint. Ulnar shortening by $2.5 \mathrm{~mm}$ to a neutral ulnar variance has been shown to reliably reduce the axial load transferred by the proximal carpal row to the distal ulna, from 42 to $18 \%$ [10]. The advantage is that the procedure remains extra-articular when compared to other surgical techniques such as distal ulnar excision, ulnar recession and the Wafer procedure [11-13]. It maintains the integrity of the distal radioulnar joint (DRUJ) and its surrounding ligaments and joint capsule, although the mechanics of the DRUJ may be altered [14].

Numerous techniques for ulnar shortening osteotomy have been described in the literature. Traditionally, a transverse freehand resection has been described to achieve the desired shortening of the ulna [6]. However, oblique osteotomies were introduced to increase the bony contact area for healing, improve compression at the osteotomy site and reduce healing time $[15,16]$. More recently osteotomy techniques using devices which allow precise width of bone resection have been shown to improve the reproducibility, speed and ease of surgery [16]. As a result, oblique osteotomy performed with the aid of a jig has become popular and has been favourably 
reported in the literature $[9,16,17]$. Despite the evolution of surgical techniques, reported complication rates for nonunion and refracture are variable and have ranged from 0 to $4 \%$ for each of the methods. Metalwork removal rates vary from 0 to $45 \%$ (Table 1).

This study reports the technique and complications over a 10 year period of performing ulnar shortening with an oblique osteotomy cut with the aid of a commercially available jig.

\section{Materials and Methods}

All patients undergoing ulnar shortening osteotomy between 2001 and 2011, 63 consecutive cases of ulnar shortening osteotomy were performed in 60 patients using the described technique. There were 37 women and 23 men with an average age of 46 years (range 14-75, SD - 13). Case-notes were reviewed retrospectively to record: demographic data; preoperative symptoms; aetiology of the ulnar-sided wrist pain; intra-operative details; bony union; and complications. The average follow-up was 20 months (1-97, SD - 19). Bivariate analysis was made using Fisher's exact test. Statistical significance was taken at the $p<0.05$ level.

Patients were seen preoperatively by the senior author (MAC) and a clinical diagnosis of UIS was made on the basis of a strong history of localised ulna sided wrist pain, combined with decreased range of wrist motion or decreased grip strength. Physical examination included the ulnocarpal stress test as described by Nakamura, in which pain is elicited when the ulna is held and the wrist is deviated ulna-wards and the forearm supinated under an axial load [26]. Radiographs were taken pre-operatively with the shoulder positioned in $90^{\circ}$ of abduction and the elbow flexed to $90^{\circ}$. The forearm was positioned in mid-pronation. Ulnar variance was assessed using the method of perpendiculars whereby the interval between a line drawn perpendicular to the long axis of the radius at its distal ulnar aspect and a parallel line marking the distal limit of the ulna were measured [25]. If the diagnosis of UIS was in doubt, patients underwent preoperative magnetic resonance imaging (MRI). If there was evidence of a TFCC tear or, if there was no clear radiographic evidence of UIS, a wrist arthroscopy was performed prior to, or at the time of, ulnar shortening osteotomy.

\section{Operative Technique}

A skin incision was made over the subcutaneous border of the ulna, protecting the dorsal sensory branch of the ulna nerve. The interval between extensor carpi ulnaris and flexor carpi ulnaris was identified and the periosteum elevated to allow plate placement. The Osteotec Ulna Shortening System cutting jig (Osteotec Ltd., Christchurch, Dorset, UK) was centred

Table 1 Summary of common complications from current literature

\begin{tabular}{|c|c|c|c|c|c|c|}
\hline & Wrists & Osteotomy & Implant & Plate Removal & Refracture & Non-Union \\
\hline Sunil et al. [17] & 97 & $\begin{array}{l}\text { Transverse freehand(45) vs } \\
\text { Oblique Rayhack(52) }\end{array}$ & $\begin{array}{c}\text { 3.5DCP 5/6hole } \\
\text { vs Rayhack }\end{array}$ & $8(8 \%)$ & $\begin{array}{l}3 \text { (freehand) } \\
1(\mathrm{jig}) \\
(4 \%)\end{array}$ & 3 (freehand) $(3 \%)$ \\
\hline Pomerance [18] & 40 & Rayhack vs Labosky single & Rayhack vs 3.5DCP & $14(35 \%)$ & $1(3 \%)$ & 0 \\
\hline Mizuseki et al. [19] & 24 & Oblique, new jig & 3.5DCP;5hole & $6(25 \%)$ & $1(4 \%)$ & 0 \\
\hline Wehbe and Cautilli [20] & 24 & AO small fragment distractor & 2.7DCP & $24(100 \%)$ & $1(4 \%)$ & 0 \\
\hline Horn [21] & 25 & LUSO screws oblique & 3.5 screws 4 & $1(4 \%)$ & $1(4 \%)$ & 0 \\
\hline Loh et al. [22] & 23 & Freehand oblique & 3.5DCP;6hole & $7(30 \%)$ & 0 (not reported) & $1(4 \%)$ \\
\hline Chen and Wolfe [2] & 18 & AO compression, oblique & 3.5DCP;6hole & $8(44 \%)$ & 0 (not reported) & 0 \\
\hline Darlis et al. [23] & 29 & Freehand stepcut & 3.5DCP;7hole & $3(10 \%)$ & 0 & 0 \\
\hline Constantine et al. [24] & 11 & Transverse, freehand & 3.5DCP;6hole & $5(45 \%)$ & 0 & 0 \\
\hline Baek et al. [25] & 31 & Transverse, freehand & $\begin{array}{l}3.5 \mathrm{DCP} ; 6 / 7 \text { hole } \\
\text { or } 3.5 \mathrm{TTP}\end{array}$ & Not reported & Not reported & 0 \\
\hline Rayhack et al. [16] & 23 & $\begin{array}{l}\text { Transverse freehand (23) } \\
\text { Rayhack jig oblique(15) }\end{array}$ & Rayhack & Not reported & Not reported & 1(radiographic) \\
\hline Tatebe et al. [4] & 6 & Transverse freehand & $3.5 \mathrm{TTP}$ & 0 & 0 & 0 \\
\hline Minami and Kato [7] & 25 & Transverse freehand & 3.5DCP;6hole & Most & $2(8 \%)$ & 0 \\
\hline Chun \& Palmer [1] & 30 & Oblique freehand & 3.5DCP;6hole & Not reported & Not reported & 0 \\
\hline Köppel et al. [9] & 47 & $\begin{array}{l}\text { Freehand transverse(32) vs } \\
\text { Freehand Oblique(17) }\end{array}$ & 2.7/3.5DCP; 6hole & $35(74 \%)$ & Not reported & $\begin{array}{l}5 \text { (transverse) }(15 \%) \\
1 \text { (oblique) } \\
(6 \%)\end{array}$ \\
\hline Hulsizer et al. [6] & 13 & Freehand transverse & 3.5DCP;6hole & $2(15 \%)$ & $1(8 \%)$ & $1(8 \%)$ \\
\hline Trumble et al. [8] & 21 & Freehand oblique & 3.5DCP;7hole & 0 & 0 & 0 \\
\hline
\end{tabular}


at the planned osteotomy site over the dorsoulnar border of the ulna and fixed proximally and distally using cortical screws. An oblique osteotomy was made using the cutting guides on the jig to resect a wafer of bone between 2.5 and $3.5 \mathrm{~mm}$ (Fig. 1), with the aim of reducing ulnar variance to just beyond neutral wherever possible. If the ulnar variance was neutral, a more conservative cut was made using the $2.5 \mathrm{~mm}$ jig. In Madelung deformities, shortening was performed to offload the ulna and to restore joint congruency to improve range of movement. Irrigation was used to minimise the effects of overheating when performing the osteotomy using the oscillating saw. The cutting jig was removed along with the resected bone (Fig. 2). A Dynamic Compression Plate (DCP) (Synthes Ltd., Welwyn Garden City, UK) was used in all but one case (61 five-hole $3.5 \mathrm{~mm}$ DCP and one eighthole $3.5 \mathrm{~mm} \mathrm{DCP}$ ). This was applied to the ulnar border of the ulna and fixed proximally and distally using the pre-drilled holes (Fig. 3). A titanium Reconstruction Plate (Synthes Ltd., Welwyn Garden City, UK) had been used in the other case as the patient reported a nickel allergy. The cutting jig is designed to compress the osteotomy site and maintain the normal rotation of the ulna once the distal and proximal cortical screws are applied. The orientation of the osteotomy also allows a single hole to be used in lag screw mode to maintain compression at the osteotomy site. This mode of fixation was used in all cases. A below elbow splint was applied for 6 weeks post-operatively.

\section{Postoperative Radiographic Assessment}

Radiographs were performed postoperatively at 6 weeks and then serially to assess for bony union or evidence of loosening. Radiographic union was defined by trabeculation across the osteotomy site, disappearance of the osteotomy gap and an absence of adverse signs such as displacement at the osteotomy site and implant loosening. If loosening or displacement was noted, union was subsequently defined as callus bridging three out of four cortices on two views. Nonunion was diagnosed on the basis of a persistent gap at the osteotomy site and failure to demonstrate progression of healing on serial radiographs beyond 6 months.

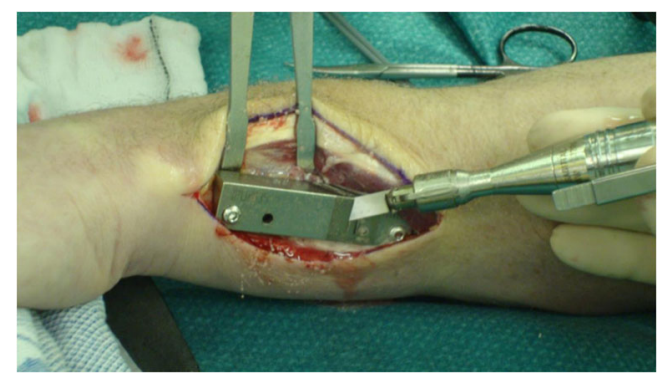

Fig. 1 Osteotec Ulna Shortening System cutting jig

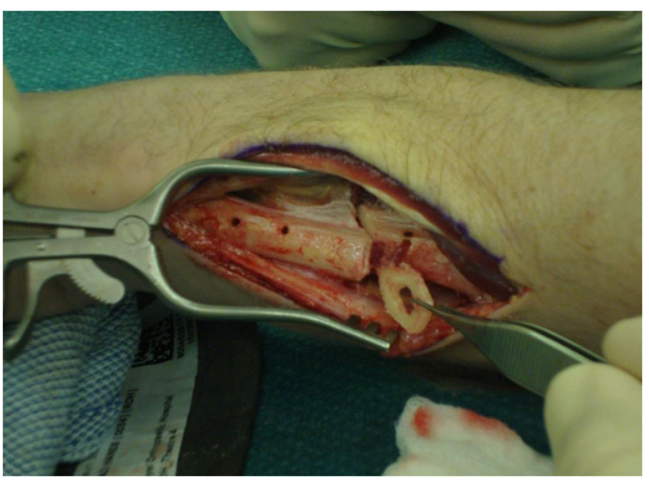

Fig. 2 Jig and resected wafer of bone removed

\section{Results}

The majority were carried out for idiopathic UIS (49 out of 63). Other reasons for performing ulnar shortening osteotomy included nine patients with ulnocarpal impaction after distal radius fracture malunion, four distal radius growth arrests from childhood injuries, one secondary to polio (Table 2).

All patients pre-operatively demonstrated neutral or positive ulnar variance on radiographs - mean $2.3 \mathrm{~mm}$ (range -0 to $10, \mathrm{SD}-2.0$, median 2), which improved post-operatively with a mean ulnar variance of $-0.2 \mathrm{~mm}$ (range - 3.0-8.0, SD - 2.0, median - 1).

Thirty-six patients $(57 \%)$ had pre-operative MRI scans of the wrist to assess for TFCC tears. Thirty-eight patients $(60 \%)$ underwent arthroscopy of the wrist prior to ulnar shortening osteotomy. Of those, $32(51 \%)$ were performed as part of the same surgical procedure. Twenty-one TFCC tears were recorded on arthroscopy or MRI, 16 of which were central, degenerative tears and five peripherally located tears/detachments.

\section{Union Rates}

Radiographic union was diagnosed in 59 cases (94\%). The median time to union for these patients was 12 weeks (range 4-78). Of these, 10 (17\%) patients took over 6 months to achieve union and were defined as delayed unions.

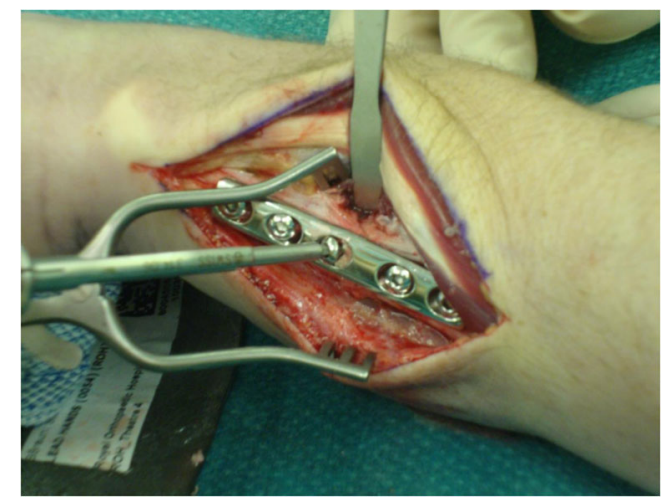

Fig. 3 Direct compression plate applied with lag screw using same drill holes 
Table 2 Demographics of patient cohort and complications

\begin{tabular}{|c|c|c|c|c|c|c|}
\hline & UIS & Previous Fracture & Growth Arrest & Other & Total & Significance (p-value) \\
\hline Number & 49 & 9 & 4 & 1 & 63 & - \\
\hline $\begin{array}{l}\text { Age (years) } \\
\text { Mean (range, SD) }\end{array}$ & $\begin{array}{l}46 \\
(26-67,11)\end{array}$ & $\begin{array}{l}48 \\
(29-75,16)\end{array}$ & 22 & 65 & 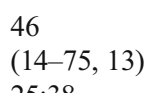 & $n s$ \\
\hline 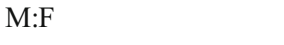 & $23: 26$ & $0: 9$ & $1: 3$ & $1: 0$ & $25: 38$ & - \\
\hline $\begin{array}{l}\text { Length of FU (months) } \\
\text { Mean (range, SD) }\end{array}$ & $15(7-28)$ & $13(7-30)$ & $12(5-26)$ & 6 & $15(7-28)$ & $n s$ \\
\hline $\begin{array}{l}\text { Time to union (weeks) } \\
\text { Median (IQR) } \\
\text { Complications }\end{array}$ & $12(6-21)$ & $7(6-12)$ & $18(14-24)$ & 10 & $12(6-21)$ & $n s$ \\
\hline Nonunion & 4 & 0 & 0 & 0 & $4(6 \%)$ & \\
\hline Refracture & 1 & 0 & 0 & 0 & $1(2 \%)$ & \\
\hline Metalwork Irritation & 26 & 4 & 2 & 0 & $32(51 \%)$ & \\
\hline
\end{tabular}

There were four nonunions (6.3\%). In three of the cases, radiographic features were consistent with loosening of the metalwork and subsequent hypertrophic nonunion. These patients had revision surgery at which stage the nonunion/partial union was confirmed, excised and stabilised using a longer (7, 8 , or 9-hole) $2.7 \mathrm{~mm}$ DCP. The use of a different calibre plate allowed for new screw holes to be used in preference to preexisting screw holes. Demineralised bone matrix (DBX, Synthes GmbH, Switzerland) was inserted into the nonunion site. The average time to revision surgery was 20 months (11, 12 and 38 months). All three cases were followed up for an average of 42 months $(15,39$ and 97 months) and progressed onto radiographic union at a median of 4 months post revision (4, 4 and 21 months). One patient had residual wrist pain and is awaiting further investigation for clicking in the DRUJ.

In the other case, the patient was listed for metalwork removal for probable metalwork irritation as the cause of pain at a mean of 13 months post index surgery.

The nonunion was noted at the time of surgery and a decision was made to treat the patient in plaster of Paris. This went onto clinical and radiological union at 12 weeks.

\section{Refracture}

There was one case of refracture following plate removal in a 29 year-old female. Radiographically, she had progressed onto rapid union but was troubled by discomfort over the plate. The plate was removed 11 months post index surgery with intra-operative evidence of union at the osteotomy site. She re-presented to clinic with pain at the osteotomy site post-operatively. Radiographs confirmed the refracture at 4 months post plate removal. There was no history of trauma prior to refracture. This was initially managed conservatively but went onto hypertrophic non-union, which was confirmed on computerised tomography. Definitive management was with a 6-hole locking plate with iliac crest bone graft. This went onto union at 8 weeks although the patient is still having significant pain and is undergoing further investigation.

\section{Metalwork Irritation}

Persistent discomfort was a common finding postoperatively in 32 out of 63 patients $(51 \%)$ although it is difficult to quantify the contribution of the metalwork irritation to the symptoms. In $27(43 \%)$ cases, plate removal was performed at the request of the patient. An additional three patients declined further surgery and in one patient the pain settled after a diagnostic injection. The mean time to plate removal was 16 months (range 9-24, SD - 4, median 19). Two patients had residual ulnar sided pain of unknown cause and were treated conservatively. Symptoms settled in 30 out of 32 patients and required no further treatment.

\section{Other Complications}

There were no cases of deep infection or neurological deficit. There was no radiological evidence of DRUJ osteoarthritis although one patient complained of post operative stiffness, but declined metalwork removal. There was one case of complex regional pain syndrome (CRPS) that occurred 2 months post-operatively in a 43 year old female. The patient was left with some residual stiffness and discomfort at 9 months which was treated conservatively.

A small proportion of patients developed other wrist problems including radiocarpal osteoarthritis (one patient treated with neurectomy), pisotriquetral osteoarthritis (one patient - treated with injection) and wrist instability (one patient - treated with wrist fusion). 


\section{Discussion}

Ulnar shortening osteotomy has been shown to provide significant improvements in pain and function. However, little attention is drawn to the risks and complications of ulnar shortening osteotomy. Freehand techniques have been associated with longer healing times and higher incidence of nonunion [17]. They are also more prone to pitfalls such as errors of angulation or rotation, overzealous or inadequate excision, and poor bony contact of the osteotomy surfaces [19]. Technical tips have been described to prevent angulation or rotational deformity and make compression easier [1]. However this does not address the problems of parallelism and width excision that can be accounted for when using a jig technique.

Although Hulsizer reported that 12 out of 13 patients achieved complete pain relief at a mean of 2.3 years with freehand transverse osteotomies, it was noted that four out of 13 had postoperative complications [6]. Chun and Palmer described an oblique freehand technique in a series of 30 cases with good results and encountered few complications, and no nonunions [1].

Outcomes and complication rates have been well documented in the literature comparing transverse and oblique as well as freehand and jig assisted ulnar osteotomies. Table 1 summaries the current cohorts showing that complication rates are low and largely independent on the type of osteotomy used. The main focus of these studies is on the theoretical advantages of assistive devices and the orientation of the osteotomy.

Rayhack et al. introduced the precision oblique osteotomy guide that allows more accurate excision. A specialised plate and compression device was then applied to achieve maximum compression at the osteotomy site with a reported a mean union time of 11 weeks and no incidence of delayed or nonunion [16]. Labosky et al. introduced a double blade technique to theoretically produce accurate ulnar shortening osteotomy [15]. Unfortunately this technique does not address the issue of compression at the osteotomy site. Horn used the Long Ulnar Sliding Osteotomy (LUSO) jig and secured the osteotomy site with interfragmentary lag screws [21]. Although there were no cases of delayed or non-unions in his findings, one would question the stability of fixation and the amount of time required to protect the osteotomy site until union is achieved. Assistive devices provide additional benefits for such osteotomies, but these devices are often expensive and often are only used with specialised plates.

The incidence of nonunion or delayed union has been reported as higher in transverse osteotomies when compared to oblique osteotomies. Rayhack et al. demonstrated this in their study, when they found delayed unions in their transverse osteotomy series during preliminary review. Transverse osteotomies were then abandoned in their cohort [16]. The theoretical advantage of oblique osteotomies may be attributed to the increase in surface area achieved by this technique. Additionally, the lag screw increases interfragmentary compression. According to the AO Foundation, interfragmentary lag screws provide 2 to $4 \mathrm{kN}$ of compression when compared to Dynamic Compression Plates (DCP) which produce $0.6 \mathrm{kN}$ to $1.0 \mathrm{kN}$ of compression [27]. The system we used enabled precise parallel osteotomies. Compression was achieved with the DCP augmented with a lag screw. Despite the theoretical advantages of the described technique, our cohort reported 11 delayed unions and four nonunions. This is contradictory to the current literature on oblique ulnar shortening (jig assisted) osteotomies and their purported advantages over freehand or transverse osteotomies.

Following ulnar shortening osteotomy, most techniques place their plates on the dorsal or ulnar surface of the ulna as this area is easily exposed. Unfortunately the drawback of such technique is the high incidence of plate irritation and removal. In our cohort, the incidence of plate removal was $45 \%$, which is higher than the reported rates in the current literature. There are three theoretical ways to address this issue - to place the plate on the volar aspect of the ulna, to use a thinner plate, or to perform the osteotomy more proximally where there is greater soft tissue coverage. However, it would be more technically challenging to place the osteotomy jig on ulnovolar surface because of its size, and thinner plates may compromise the stability of the osteotomy fixation. More proximal osteotomies have not been described in the literature but would theoretically involve a more extensive exposure.

Refracture has been reported in the literature but has been associated with further trauma after plate removal. We had one refracture with no associated trauma after plate removal at 11 months. Biomechanically, plates represent a load-bearing implant and as a result, affects the strength and structure of bone underneath the plate [18]. It has been postulated that stress shielding from the plate is responsible for lost of bone mineral density. Rosson et al. estimates that it takes 2 years for cortical atrophy under the plate to return to its pre-fracture strength after plate removal [28]. Malone et al. also showed that microfractures occur around screw holes when they are removed from tubular bones. These sites represent a stress riser for fractures to occur and propagate with sufficient trauma [29]. It is apparent that the greatest risk of refracture is soon after metalwork has been removed. According to Sunil and Pomerance, metalwork should only be removed once the osteotomy has healed on sequential radiographs $[17,18]$. In our series, we found that oblique osteotomies in particular can give an impression of cortical and trabecular continuity across the osteotomy. If the angle of the $\mathrm{x}$-ray beam is not in line with the osteotomy cut, this will produce an oblique film with overlap of the two osteotomy surfaces. In addition, the precise cut and realignment from the described technique can make 
assessing radiographic union particularly difficult as the bone heals by primary bone healing. Trabeculation across the fracture site is often subtle and the plate often obscures the view on plain radiographs. This can lead to the conclusion that the osteotomy has united [17]. These factors may contribute to our refracture rate particularly if there has been a delayed union that has been missed. We therefore recommend that plates should not be removed until at least 12 months after initial fixation to avoid destabilising a delayed union.

In summary, oblique ulnar osteotomy using a jig technique is consistently reliable and reproducible in shortening the ulna and maintaining alignment, rotation and compression at the osteotomy site. We have shown that, in the majority of patients, symptoms improved although a small proportion $(3.2 \%)$ had residual pain after metalwork removal. However, it would appear that the morbidity associated with the procedure may be underestimated as highlighted by our complication rates $-6.3 \%$ risk of nonunion, $1.6 \%$ of refracture and, $45 \%$ of patients requiring plate removal. Surgeons should consider this and counsel patients appropriately when offering ulnar shortening osteotomy, in particular the likelihood of the need for plate removal.

Compliance with Ethical Standards: There was no funding for this study.

Ethical Approval All procedures performed in studies involving human participants were in accordance with the ethical standards of the institutional and/or national research committee and with the 1964 Helsinki declaration and its later amendments or comparable ethical standards.

For this type of study formal consent is not required.

Conflict of Interest Statement SKL Chan declares that he has no conflict of interest.

T Singh declares that he has no conflict of interest.

$\mathrm{R}$ Pinder declares that he has no conflict of interest.

$\mathrm{S}$ Tan declares that he has no conflict of interest.

MA Craigen declares that he has no conflict of interest.

\section{References}

1. Chun S, Palmer AK (1993) The ulnar impaction syndrome: follow-up of ulnar shortening osteotomy. J Hand Surg Am 18:46-53. doi:10.1016/0363-5023(93)90243-V

2. Chen NC, Wolfe SW (2003) Ulna shortening osteotomy using a compression device. J Hand Surg Am 28:88-93. doi:10.1053/jhsu. 2003.50003

3. Minami A, Ogino T, Minami M (1987) Treatment of distal radioulnar disorders. J Hand Surg Am 12:189-196. doi:10.1016/ S0363-5023(87)80269-1

4. Tatebe M, Nakamura R, Horii E, Nakao E (2005) Results of ulnar shortening osteotomy for ulnocarpal impaction syndrome in wrists with neutral or negative ulnar variance. J Hand Surg Br 30:129_ 132. doi:10.1016/j.jhsb.2004.09.005
5. Boulas HJ, Milek MA (1990) Ulnar shortening for tears of the triangular fibrocartilaginous complex. J Hand Surg Am 15:415420. doi:10.1016/0363-5023(90)90052-S

6. Hulsizer D, Weiss AP, Akelman E (1997) Ulna-shortening osteotomy after failed arthroscopic debridement of the triangular fibrocartilage complex. J Hand Surg Am 22:694-698. doi:10.1016/ S0363-5023(97)80130-X

7. Minami A, Kato H (1998) Ulnar shortening for triangular fibrocartilage complex tears associated with ulnar positive variance. J Hand Surg Am 23:904-908. doi:10.1016/S0363-5023(98)80171-8

8. Trumble TE, Gilbert M, Vedder N (1997) Ulnar shortening combined with arthroscopic repairs in the delayed management of triangular fibrocartilage complex tears. J Hand Surg Am 22:807-813. doi:10.1016/S0363-5023(97)80073-1

9. Köppel M, Hargreaves IC, Herbert TJ (1997) Ulnar shortening osteotomy for ulnar carpal instability and ulnar carpal impaction. J Hand Surg Br 22:451-456. doi:10.1016/S0266-7681(97)80265-3

10. Palmer AK (1987) The distal radioulnar joint. Anatomy, biomechanics, and triangular fibrocartilage complex abnormalities. Hand Clin 3:31-40

11. Darrach W (1913) Partial excision of lower shaft of ulna for deformity following colles's fracture. Ann Surg 57:764-765

12. Darrow Jr JC, Linscheid RL, Dobyns JH, Mann 3rd JM, Wood MB, Beckenbaugh RD (1985) Distal ulnar recession for disorders of the distal radioulnar joint. J Hand Surg Am 10:482-491. doi:10.1016/S0363-5023(85)80069-1

13. Feldon P, Terrono AL, Belsky MR (1992) Wafer distal ulna resection for triangular fibrocartilage tears and/or ulna impaction syndrome. J Hand Surg Am 17:731-737. doi:10.1016/0363-5023(92)90325-J

14. Thiru RG, Ferlic DC, Clayton ML, McClure DC (1986) Arterial anatomy of the triangular fibrocartilage of the wrist and its surgical significance. J Hand Surg Am 11:258-263. doi:10.1016/S03635023(86)80065-X

15. Labosky DA, Waggy CA (1996) Oblique ulnar shortening osteotomy by a single saw cut. J Hand Surg Am 21:48-59. doi: 10.1016/S0363-5023(96)80153-5

16. Rayhack JM, Gasser SI, Latta LL, Ouellette EA, Milne EL (1993) Precision oblique osteotomy for shortening of the ulna. J Hand Surg Am 18:908-918. doi:10.1016/0363-5023(93)90065-B

17. Sunil TM, Wolff TW, Scheker LR, McCabe SJ, Gupta A (2006) A comparative study of ulnar-shortening osteotomy by the freehand technique versus the rayhack technique. J Hand Surg Am 31:252257. doi:10.1016/j.jhsa.2005.09.017

18. Pomerance J (2005) Plate removal after ulnar-shortening osteotomy. J Hand Surg Am 30:949-953. doi:10.1016/j.jhsa.2005.06.005

19. Mizuseki T, Tsuge K, Ikuta Y (2001) Precise ulna-shortening osteotomy with a new device. J Hand Surg Am 26:931-939. doi: 10.1053/jhsu.2001.26201

20. Wehbe MA, Cautilli DA (1995) Ulnar shortening using the AO small distractor. J Hand Surg Am 20:959-964. doi:10.1016/ S0363-5023(05)80143-1

21. Horn PC (2004) The long ulnar sliding osteotomy. J Hand Surg Am 29:871-876. doi:10.1016/j.jhsa.2004.04.024

22. Loh YC, Van Den Abbeele K, Stanley JK, et al (1999) The results of ulnar shortening for ulnar impaction syndrome. J Hand Surg Br 24:316-320. doi:10.1054/jhsb.1999.0062

23. Darlis NA, Ferraz IC, Kaufmann RW, Sotereanos DG (2005) Stepcut distal ulnar-shortening osteotomy. J Hand Surg Am 30:943948. doi:10.1016/j.jhsa.2005.05.010

24. Constantine KJ, Tomaino MM, Herndon JH, Sotereanos DG (2000) Comparison of ulnar shortening osteotomy and the wafer resection procedure as treatment for ulnar impaction syndrome. J Hand Surg Am 25:55-60. doi:10.1053/jhsu.2000.jhsu025a0055

25. Baek GH, Chung MS, Lee YH, Gong HS, Lee S, Kim HH (2005) Ulnar shortening osteotomy in idiopathic ulnar impaction 
syndrome. J Bone Joint Surg Am 87:2649-2654. doi:10.2106/ JBJS.F.00320

26. Nakamura R, Horii E, Imaeda T, Nakao E, Kato H, Watanabe K (1997) The ulnocarpal stress test in the diagnosis of ulnar-sided wrist pain. J Hand Surg Br 22:719-723. doi:10.1016/S02667681(97)80432-9

27. Perren SM (1991) Basic aspects of internal fixation. In: Miller ME, Allgower M, Schneider R, Willenegger H (eds) Manual of Internal
Fixation. Techniques Recommended by the AO-ASIF Group, 3rd edn. Springer-Verlag, New York, pp. 1-96

28. Rosson JW, Petley GW, Shearer JR (1991) Bone structure after removal of internal fixation plates. J Bone Joint Surg Br 73:65-67

29. Malone CB, Heiple KG, Burstein AH (1977) Bone strength: before and after removal of unthreaded and threaded pin and screws. Clin Orthop Relat Res:259-260 